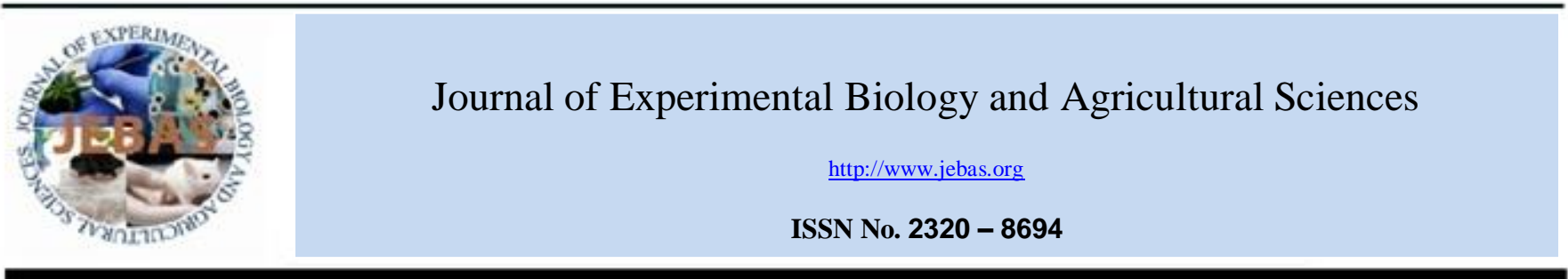

\title{
EFFECT OF ANTIGEN CONTENT ON AVIAN INFLUENZA VACCINE EFFICIENCY
}

\author{
Nouran Y. Hamed ${ }^{1}$, AhmedKandeil ${ }^{1}$, Jamal S. M. Sabir ${ }^{2,3}$, Salah E. M. Abo-Aba ${ }^{2,3,4^{*} \text {, }}$ \\ Nahid H. hajrah ${ }^{2,3}$, Mohammad K. Al-ghamdi ${ }^{5}$, Alawiah M. Alhibshi ${ }^{2,3}$, \\ Abdelfatteh El Omri ${ }^{2,3}$, Areej K. Al-gamdi ${ }^{5}$, Mohamed A. Ali ${ }^{1,2}$ \\ ${ }^{1}$ Center of Scientific Excellence for Influenza Viruses, National Research Center, Giza, Egypt. \\ ${ }^{2}$ Center of excellence in Bionanoscience Research, King Abdulaziz University (KAU), Jeddah 21589, Saudi Arabia. \\ ${ }^{3}$ Biotechnology Research Group, Department of Biological Sciences, Faculty of Science, King Abdulaziz University (KAU), Jeddah 21589, Saudi Arabia. \\ ${ }^{4}$ Microbial Genetics Department, Genetic Engineering and Biotechnology Division, National Research Center, Dokki, Giza, Egypt. \\ ${ }^{5}$ Department of Physics, Faculty of Science, Universityof Jeddah, Saudi Arabia
}

Received - October 29, 2018; Revision - December 08, 2018; Accepted - December 14, 2018

Available Online - December 15, 2018

DOI: http://dx.doi.org/10.18006/2018.6(6).997.1003

\section{KEYWORDS}

H5N1

Vaccine

Antigen content

Vaccination

HA

Immunogenicity

\begin{abstract}
Many avian influenza vaccines are currently used to treat avian influenza virus's infection in many countries of Middle East. Information about the role of antigen content in vaccine efficiency is vital for the choice of ideal vaccines and vaccination programs, in addition to licensing of new avian influenza (AI) vaccines. In present work, we studied the serological response of SPF chicken towards 7 different concentrations of avian influenza H5N1 virus (8HA, 16HA, 32HA, 64HA, 128HA, 256HA, 512 HA). Different concentrations of the vaccine strain of H5N1 virus were prepared and mixed with an adjuvant to prepare experimental vaccines with different concentrations of antigen content. Serological responses of post vaccinated chickens were monitored over the course of 6 weeks. After calculation of hemagglutination inhibition (HI) values for each group to determine the antibody titers, it was found that the maximum antibody levels were produced by antigen contents 512 and 256 HAU. Results of the present study indicated that low antigenic groups have promising protective effect.
\end{abstract}

* Corresponding author

E-mail: salah_aboaba@yahoo.com (Salah E. M. Abo-Aba)

Peer review under responsibility of Journal of Experimental Biology and Agricultural Sciences.

Production and Hosting by Horizon Publisher India [HPI] (http://www.horizonpublisherindia.in/).

All rights reserved.
All the article published by Journal of Experimental Biology and Agricultural Sciences is licensed under a Creative Commons Attribution-NonCommercial 4.0 International License Based on a work at www.jebas.org.

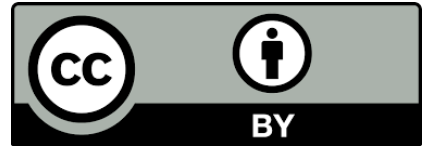




\section{Introduction}

Influenza viruses are highly contagious viral infection that infects humans of all ages along with animals. Annual epidemics of influenza infection might cause significant morbidity and mortality, which imposing a substantial economic burden. Many public health officials feel that the next pandemic may be imminent, and efforts to minimize its impact are crucial (Kamradt-Scott, 2012). Increasing the availability of in-house vaccine strain, lowering the prices could encouraging poultry farmers to vaccinate their animals. Pathogenesis of HPAIV is detonated to its ability to reproduce inside the body's internal tissues and organs which caused internal organ failure and ultimately leading to death. While in case of LPAIVs, mild or symptom based infection was reported (Rott, 1992). One of the most important control measures was vaccination strategy. From 2006 onward, many commercially available H5 vaccines were licensed to be uses in poultry farms in different countries. Initially commercial vaccines showed variable immune reactivity. However, it declined as the virus continued to mutate. The variability is due to the incompatibility of the commercial viral vaccine strain and domestic poultry (Kayali et al., 2016). Therefore, local manufacturing of vaccine strains compatible with domestic strain is crucial. Nonetheless, vaccine manufacturing is not an easy feat, making it extremely expensive for the in-house factories to create with the inability of producing in large quantity that would cover the local demand. The antigen content of avian influenza virus vaccine is essential to stimulate antibody response and reach protective antibody titers (Swayne et al., 2015). In this study, avian influenza vaccines having different antigen contents were tested in SPF chickens to determine the highest protective antibody titers generated from the lowest concentration of antigen content.

\section{Materials and Methods}

\subsection{Virus}

The pathogenicity of an AI A/chicken/Egypt/ S10552B /2015(H5N1) virus was previously modified (Kandeil et al., 2017) by altering the multiple basic amino acids sequence (RRKKR) at the cleavage site of the HA accession Number (MG662416.1) to create the low pathogenic monobasic sequence (R) using plasmidbased reverse genetics (Webby et al., 2004).

Avian influenza virus was propagated in 9-11 day old specific pathogen free (SPF) embryonated chicken eggs. Briefly, the low pathogenic AI H5N1 vaccine strain was diluted in egg infection medium (1X PBS and 2\% antibiotic antimycotic) and was inoculated into the allantoic cavity of 10 days old SPF eggs then incubated at $37^{\circ} \mathrm{C}$ for $48 \mathrm{hrs}$. Infected eggs after $48 \mathrm{hrs}$ were chilled at $4^{\circ} \mathrm{C}$ before being harvested. The harvested allantoic fluid was stored immediately at $4^{\circ} \mathrm{C}$.

\subsection{Virus titration}

\subsubsection{HA assay}

The harvested virus was titrated by hemaagglutination assay (HA) according to WHO protocol (WHO, 2002). Briefly, $50 \mu 1$ volumes of PBS were aliquoted in a 96 well U-shaped plate (Greiner BioOne, Germany). Then $50 \mu 1$ of virus was added to the first well and two fold serial dilutions were made across the plate. Finally, $50 \mu 1$ of $0.5 \%$ chicken RBCs were added to all wells and the plate was shaken to ensure mixing. The plate was incubated for $30 \mathrm{~min}$ at room temperature before being examined for HA titer. The HA titer of virus was calculated as the reciprocal of the highest dilution of the virus that caused complete agglutination of chicken RBCs

\subsubsection{Egg Infection Dose 50 (EID50):}

EID50 is the amount of virus that infects 50 percent of inoculated eggs. A series of ten-fold dilutions are carried out on the viruses. Then, 10-day-old SPF embryonated chicken eggs were inoculated with $100 \mu$ of different dilutions of each virus (3 eggs for each dilution). Inoculated eggs were incubated for 2 days in $37^{\circ} \mathrm{C}$ then chilled at $4^{\circ} \mathrm{C}$ for $4 \mathrm{hrs}$. Allantoic fluids were subjected to HA assay. EID50 of each virus was then calculated using by Reed and Muench method (Reed \& Muench, 1938). The infectivity titer is expressed as EID50 per $\mathrm{mL}$.

\subsection{Vaccine preparation}

Seven different concentrations of the low pathogenic $\mathrm{rgA} /$ chicken/Egypt/S10552B /2015(H5N1) virus ranged from HA $=8$ to $\mathrm{HA}=512$ were prepared. Each concentration of viral antigen was titrated by EID50. Inactivation of AI virus at different concentrations was carried out using formalin in a final concentration of $0.1 \%$ of the total volume. For vaccine formulation, different concentrations of virus antigens were mixed with Montanide ISA 70 VG (Seppic, France) in the ratio recommended by the manufacturer ( 30 antigen/70 oil). This mixture was homogenized for $3 \mathrm{~min}$ in ice bath, and then the mixture was left to settle down for $5 \mathrm{~min}$ then mixed again for $3 \mathrm{~min}$.

\subsection{Bird immunization}

Two weeks old eighty SPF (white leghorn) chickens were used in this immunization study. SPF chickens were brought at age 7 days and allotted into 8 groups (10 birds/group). Sera collected from 10 randomly selected chicks were tested for H5N1 antibodies resulting from maternal immunity. Using $\mathrm{HI}$ assay, the antibody titer against $\mathrm{H} 5 \mathrm{~N} 1$ virus was monitored and corresponding Log2 titer was calculated (WHO, 2002). At the age of 3 weeks, birds in groups 1 to 7 were vaccinated via the intramuscular (thigh muscle) route with the prepared inactivated vaccines $(0.5 \mathrm{ml}$ dose of the prepared vaccine formulas). Group 8 was kept as an 
unvaccinated negative control. Serum samples were collected on a weekly basis for 6 week post vaccination. The hemaagglutination inhibition (HI) test was carried out to monitor the post vaccination humoral immune response for each vaccine formula using the homologous antigen.

\subsection{Hemagglutination inhibition (HI) assay}

A standardized quantity of HA antigen (4 HA units) was mixed with serially diluted serum samples and red blood cells (RBCs) are added to detect specific binding of antibody to the HA molecule. The HI titers of collected sera were estimated against AI H5N1 virus as described previously.

\subsection{Virus concentration for protein separation}

Collected allantoic fluids were used for preparation of different concentrations of vaccine ranged from 8 to 512 HAU. Different concentrations were clarified from cell debris by centrifugation at $4500 \mathrm{rpm}$ for $15 \mathrm{~min}$. Viral particles were pelleted through a sucrose cushion. A volume of $15 \mathrm{ml}$ of the viral preparation was carefully pipetted on top of a $20 \%$ sucrose solution $(6 \mathrm{ml})$ and then centrifuged at $32,000 \mathrm{rpm}$ for $2 \mathrm{~h}$ at $4{ }^{\circ} \mathrm{C}$. The pellet was resuspended in $300 \mu 1$ Phosphate-buffered saline (PBS).

\subsection{SDS- PAGE of different virus concentrations:}

Samples were separated through 4\% stacking and $12.5 \%$ resolving gels in a Bio-Rad Mini- Protean II electrophoresis chamber (Bio-Rad Laboratories, Munich, Germany) according to Laemmli (1970). Diluted samples (1:1) in reducing sample buffer were loaded onto the stacking gel $(10 \mu \mathrm{l}$ of each sample/ each lane). To determine the molecular weights of the resolved protein fractions, low range molecular weight protein marker (10 to 165 $\mathrm{kDa}$, Lonza) was loaded on the same gel. Electrophoresis was performed at $80 \mathrm{mV}$ constant voltage and stopped after the bromophenol blue dye reached the end of the resolving gel. After electrophoresis, gels were stained using coomassie staining solution at $37^{\circ} \mathrm{C}$ for $2 \mathrm{hrs}$ in a shaker incubator. After staining, the gel was washed twice with $\mathrm{ddH} 2 \mathrm{O}$ to remove excess stain then covered with destaining solution. Three to four changes of destaining solution was usually sufficient to visualize blue bands in a clear background.

\subsection{Western blotting}

Western blotting techniques were done according to Towbin et al. (1979). Following electrophoresis, proteins were electrically transferred from the gel to a nitrocellulose sheet (BA85, pore size $0.45 \mathrm{~mm}$; Schleicher and Schuell, Dassel, Germany), at $6 \mathrm{~V} / \mathrm{cm}$ and $250 \mathrm{~mA}$ overnight at $4{ }^{\circ} \mathrm{C}$ in a transfer buffer. The membranes were cut into individual strips and were washed 3 times with washing buffer for $5 \mathrm{~min}$; and blocked against nonspecific binding with blocking buffer at room temperature for $1 \mathrm{hr}$. Strips were rewashed 3 times as mentioned above, and incubated with diluted primary antibody (vaccinated chicken serum; 1:50) in blocking buffer at room temperature for 2 hrs. After another 3 washes, strips were incubated for $2 \mathrm{hrs}$ at room temperature with diluted peroxidase labeled secondary antibodies (anti-chicken IgG; 1:500 in washing buffer, KPL, Gaithersburg, Germany). Developing the strips with the DAB peroxidase specific substrate allowed visualization of immune complexes on the nitrocellulose membrane. The molecular weights of the immunogenic viral peptides were determined by comparing their migration fronts to those of the molecular weight marker.

\section{Results}

3.1 Propagation and titration of AI vaccine strain in SPF embryonated chicken eggs

LPAI rg A/chicken/Egypt/S10552B /2015(H5N1) strain was propagated in 9 to 11 days old, SPF for $48 \mathrm{hrs}$ post infection. Allantoic fluid was harvested and titrated using HA assay and EID50. Using chicken red blood cells, the virus titer was $9 \log 2$ $\mathrm{HAU} / 50 \mu \mathrm{l}$. The virus was titrated using infectivity titration on SPF eggs. Using Reed and Muench mathematical method, the virus titer was calculated as $106.5 \log 10 \mathrm{EID} 50 / \mathrm{ml}$.

Based on the HA titration results, seven different concentrations of the low pathogenic (LP) A/chicken (H5N1) virus ranged from $\mathrm{HA}=512$ to $\mathrm{HA}=8$ were prepared in PBS. Each dilution was titrated in SPF eggs by EID50. The titers of viral antigens were calculated as showed in Table 1 .

Table 1 Virus titer by HAU and the corresponding EID 50

\begin{tabular}{|lc|}
\hline HAU & EID $_{50} / 0.1 \mathrm{ml}$ \\
\hline 512 & $10^{5.75}$ \\
\hline 256 & $10^{5.5}$ \\
\hline 128 & $10^{5.75}$ \\
\hline 64 & $10^{3.5}$ \\
\hline 32 & $10^{3.5}$ \\
\hline 16 & $10^{2.5}$ \\
\hline 8 & $10^{2.5}$ \\
\hline
\end{tabular}

3.2 Profiles of resolved proteins from the different prepared vaccines by SDS-PAGE:

SDS-PAGE profiles of the seven different types of papered antigen sat different concentrations were compared. Staining of the resolved proteins by coomassie stain visualized viral peptides at 81, 60, 45. 38, 27 and $25 \mathrm{kDa}$. Viral peptides at 81, 60, 45, 27 and $25 \mathrm{kDa}$ may respectively represent viral HA0, NP, HA1, M1, 
and HA2 proteins. Different types of formulated vaccine containing different concentration of viral antigen 512, 256, 128, 64, 32, 16, and $8 \mathrm{HAU}$ developed variation in intensity on SDS-PAGE based on antigen content as shown in Figure 1.

\subsection{Protein identification of the different} prepared vaccines using Western Blot:

Western blot analysis results demonstrated visualization of immunogenic peptides at nearly $81,60,45,27$ and $25 \mathrm{kDa}$ correspond to viral HA0, NP, HA1, M1, and HA2 proteins respectively using positive chicken sera. Columns representing 256 and $512 \mathrm{HAU} / 50 \mu \mathrm{L}$ showed the highest intensity which indicates that the respective animal groups are expected to show high levels of antibodies. For 128,64 , and $32 \mathrm{HAU} / 50 \mu \mathrm{L}$ columns, band were still visible and visualized, which predicts that there will be high levels of antibodies in their respective animal groups in response to vaccination. For 8 and $16 \mathrm{HAU} / 50 \mu \mathrm{L}$ columns faint band were detected indicating insignificant levels of antibodies will be produced for their respective animal groups in response to vaccination as shown in figure 2 .

\subsection{Antibody response in SPF chickens}

Sera collected from 10 randomly selected SPF chicks were tested for maternal antibody at two weeks of age cross-reacted with rgA/chicken/Egypt/S10552B /2015(H5N1) with $\log 2$ HI mean titer of 4 . At week 3 of age (day of vaccination), the maternal antibody titer dropped to $\log 2$ mean titer 1.2. Serological responses to different types of vaccines were weekly assessed till week 6 post vaccination against homologues virus. Using HI assay of chicken red blood cells, the antibody titer against H5N1 virus was monitored and corresponding $\log 2$ titer was calculated. At week 3 of age, birds in groups 1 to 7 were vaccinated via the intramuscular (thigh muscle) route with different types of inactivated vaccines (in $0.5 \mathrm{ml}$ doses of the prepared vaccine formulas). Group 8 was kept as an unvaccinated negative control. Serum samples were collected on a weekly basis for 6 week post vaccination. The hemagglutination inhibition (HI) test was carried out to monitor the post vaccination humoral immune response for each vaccine formula using the homologous A/chicken (H5N1) antigen (figure 3).

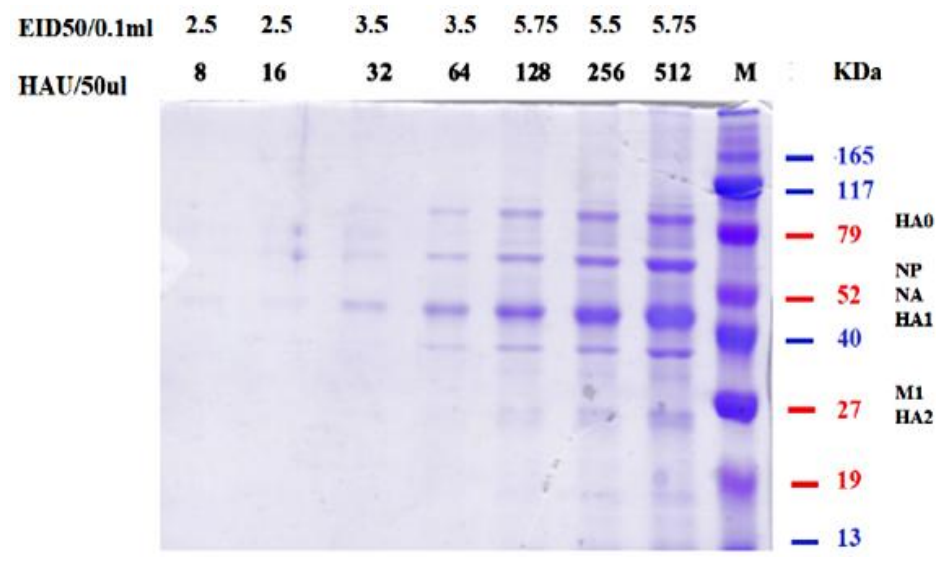

Figure 1 SDS-PAGE comparing the seven different antigen (From $R$ to L: 512, 256, $128,64,32,16,8$; staining the resolved proteins of the inactivated vaccines using Coomassie blue Stain)

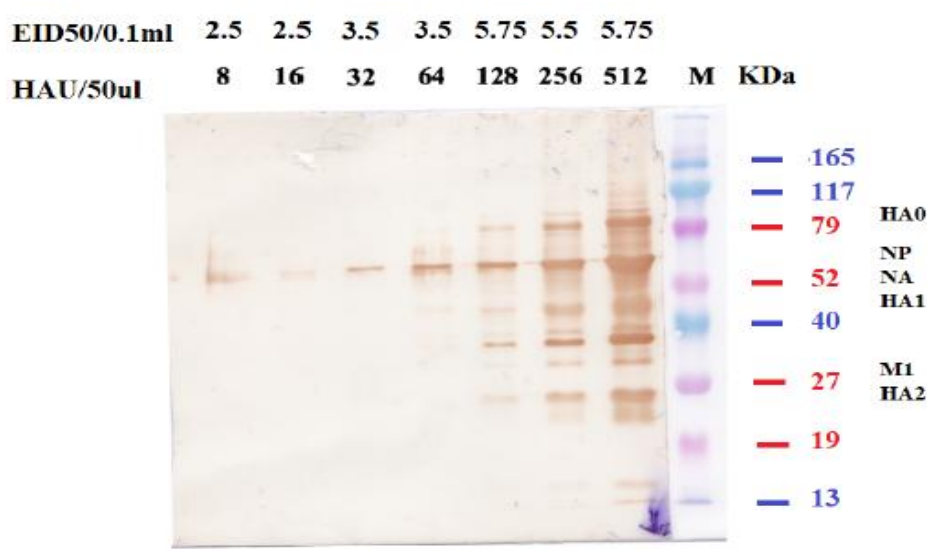

Figure 2 Western blot comparing the different viral protein concentration for the 7 different virus concentrations having antigen content ( $R$ to $L: 512,256,128,64,32$, $16,8)$, referenced to protein ladder ranged from 13 to $165 \mathrm{kDa}$.

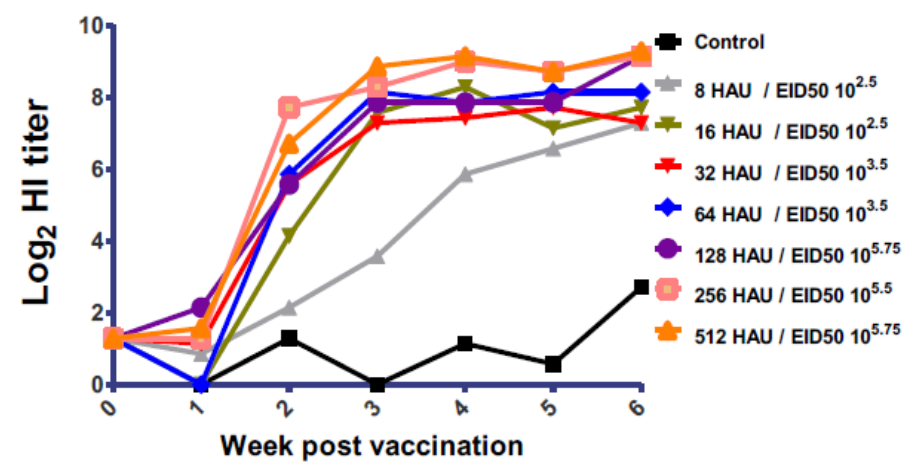

Figure 3 Follow up of immune response (HI titers) raised against different antigen content of inactivated vaccines in SPF chicken during 6 weeks post vaccination. 
The mean HI titer of all serum samples obtained before immunizing the SPF chickens at 0 to 7 days were $4 \pm \log 2$ for H5N1 specific antibodies. Comparing the kinetic of antibody titers of vaccinated chicken groups against $\mathrm{H} 5 \mathrm{~N} 1$ virus were weekly monitored using HI assay as shown in figure 3.Control group shows insignificant levels of antibodies in sera over the period of the experiment. Antibody levels start increasing after week 2. Levels of antibodies produced over the course of 6 weeks post vaccination were increased by time. The levels of antibodies were increased by increasing of antigen content as shown in Figure 3 . The maximum antibody levels were produced by antigen contents 512 and 256 HAU.

\section{Discussion}

HPAI H5N1 has persistently circulated among many poultry population in defiance to all the control measures implemented by the ministries in the world (Kim et al., 2010). Eventually, all control measures were focus on vaccination promoting it to being the in-action control plan for HPAI H5N1 spread (Kim et al., 2010; Kayali et al., 2016). Till the present time, different countries including some Arabic countries has licensed 26 types of inactivated vaccines against $\mathrm{H} 5$ to be employed in poultry farms (Kayali et al., 2016). However, due to the persistent use of vaccines coupled with the difficulty of vaccination manufacturing process; inability to cover the demands of the poultry industry. Imported commercial vaccines were later found out to not be able to prompt protection, as a result to the incompatibility of the vaccine with the domestic poultry strain (Kim et al., 2010). This is factual grounded on the case of unsuccessful actions by H5N1 inactivated vaccines that were imported from China and Europe, to provoke protection. Factors that affect vaccine efficiency can be grouped into four main categories viz., vaccine factors, host factors, human factors and environmental factors. Firstly, for vaccine factor it includes: annual revaccination, adjuvant and degree of attenuation. Secondly, host factors comprise of maternal antibody, concurrent disease, immune system function, and breed variation. Thirdly, human factor encompasses: incorrect handling or storage of vaccines and insufficient time between vaccination and exposure. Finally, environmental factors under those circumstances, systemic analyzing of all the factors that affect the vaccines' efficacy is needed. Therefore, antigen content is a main factor in determining the vaccines' efficiency and its ability to produce protection against the virus. In fact, there is shortage of literature displaying the connection between antigen content of vaccines, immunogenic responses, and the reduction in replication of viruses following challenge. For vaccine efficacy analysis, studies of dose response vaccinations are ideal to identify the vaccine antigen content and the levels of antibodies produced needed to instigate protections and prevent viral shedding (Maas et al., 2009).
It was established that antigen content defines the efficacy of inactivated avian influenza vaccine for poultry, also the viral strain plays a role in the required antigen content for the success of the vaccination process (Swayne et al., 1999). Together with, correspondence (homology) of the vaccine virus with the challenge virus (Swayne et al., 2000a; Swayne et al., 2000b). There is direct correlation between inactivated vaccines' antigen content and seroprevelance in chickens, granted that there is minimal antigenic variation between vaccines strains used with similar adjuvants (Maas et al., 2009).

Nevertheless, avian influenza viruses that have the same serological distinction demonstrate substantial antigenic disparity (Spackman et al., 2003). With that in mind, antibodies levels elevated against an avian influenza virus can display fluctuating reactions against viruses having the same serotype (Swayne et al., 2000a; Swayne et al., 2000b).

Building on HA titration results, the 7 different concentrations of the virus were prepared ranging from $\mathrm{HA}=512$ to $\mathrm{HA}=8$. SDS PAGE visualized the viral proteins of the 7 different prepared concentrations of the virus at $81,60,45,27$ and $25 \mathrm{kDa}$ which respectively represent viral $\mathrm{HA} 0, \mathrm{NP}, \mathrm{HA} 1, \mathrm{M} 1$, and HA2 proteins. Western blot identified the antibody response to the prepared 7 concentrations of the virus, consequently, giving primary indication of the vaccine concentrations that will raise the antibody levels in sera. Group 256 and 512 were intensely shown on the membrane, in addition to the significant band visibility in 32, 64, 128 groups. The degree of immunity and protection of vaccines could be implied through serological results. Mortality of birds is avoided when HI titer values are 40 or more (Liu et al., 2003) and virus replication is avoided when HI titers are above 120 (Swayne et al., 2006). However, cases have been reported where protection occurred with HI levels below 40 (Swayne et al., 2001; van der Goot et al., 2005; Kumar et al., 2007). This could be due to that HI assay is focused on HA neutralizing antibodies rather than assessing antibodies to epitope and immunogenic viral protein, for instances antibody against different parts of HA proteins (fusion domain or stalk), NS, NP, and M proteins that have the potential to be cross-reactive in birds that will eventually lead to cross-protection (van der Goot et al., 2008; Park et al., 2009). Which leads to the assumption that cellular immunity has a vital part in efficacy of AI vaccination than previously believed (Khalenkov et al., 2009). For potent vaccines, having sufficient amount of HA is crucial, however, HA content quantification is challenging using the conventional testing procedures, which quantify hemagglutin in unit, infectious titer or HA protein proceeding preparation of the final result using adjuvant. HA proteins content quantification is a very straightforward process (Wood et al., 1985), however, the process is burdensome after adjuvant preparation. In conclusion, the decreased concentration 
of $\mathrm{H} 5 \mathrm{~N} 1$ antigen content used in vaccine preparation needs to be confirmed that it resulted in complete neutralization of the viral infection particles. This is done not only by homologous challenge; heterologous challenge is a necessity. According to a study done by Maas et al. (2009) which established that elevated virustiter have the possibility to still be detectable in chickens protected against the medical disease, although that the mean virus titers intensely declined post vaccination, notable virus titer werefound in some chicken individual. The study concluded that less than optimal AIvaccine antigen dose, results in clinical protection post field challenge, however, it will not hinder virus circulation between herds neither preventing human exposure to the virus. Particularly, when there is less than optimal vaccination, and fluctuating immunity post vaccination, the virus could circulate in vaccinated herds unhindered and unnoticed. Provided that vaccination agendas directed towards deterrence or prohibition of virus circulation must thereupon constantly be supplemented with virus circulation surveillance of ground virus among vaccinated populations.

In this study, vaccines with 103.5 EID50 $/ 0.1 \mathrm{ml}$ and more were found to be immunogenic, and could be used in further analysis only if there is no mismatching, must be used against homologous strains. To confirm the protective level associated with the use of low antigen content of AI-virus at HA 32, 64, and 128 corresponding with EID50, $10^{3.5}$, and $10^{5.75}$ respectively.

\section{Acknowledgements}

This work was funded by the following: The Deanship of Scientific Research, King Abdulaziz University, Jeddah, Saudi Arabia, contract number (I-005-436); and by the Science and Technology Development Fund (STDF) in Egypt, under contract number 5175 .

\section{Author Contributions}

Ahmed Kandeil,Jamal S. M. Sabir, Nouran Y. Hamed, Salah E. M. Abo-Aba, Nahid H. hajrah and Ali MA designed the experiments, all authors performed the experiments and analyzed the data. Ahmed Kandeil, Jamal S. M. Sabir ,Salah E. M. AboAbaand Ali MA wrote the paper and revised the final manuscript. All authors reviewed the manuscript.

\section{Conflict of Interest}

The authors declare that there is no conflict of interest regarding the publication of this research paper.

\section{References}

Kamradt-Scott A (2012) Changing Perceptions: of Pandemic Influenza and Public Health Responses. American Journal of Public Health 102: 90-98.
Kandeil A, Mostafa A, El-Shesheny R, El-TaweelAN, Gomaa M, Galal H, Kayali, G, Ali MA (2017) Avian influenza H5N1 vaccination efficacy in Egyptian backyard poultry. Vaccine 35: 6195-6201.

Kayali G, Kandeil A, El-Shesheny R, Kayed AS, Maatouq AM, Cai Z, Ali MA (2016) Avian Influenza A(H5N1) Virus in Egypt. Emerging Infectious Diseases 22: 379-388. DOI: http://doi.org/10.3201/eid2203.150593.

Khalenkov A, Perk S, Panshin A, Golender N, Webster RG (2009)Modulation of the severity of highly pathogenic H5N1 influenza in chickens previously inoculated with Israeli H9N2 influenza viruses. Virology 383: 32-38.

Kim JK, Kayali G, Walker D, Forrest HL, Ellebedy AH, Griffin YS, Aldridge JR (2010) Puzzling inefficiency of H5N1 influenza vaccines in Egyptianpoultry. Proceedings of the National Academy of Sciences 107: 11044-11049.

Kumar M, Chu HJ, Rodenberg J, Krauss S, Webster RG (2007) Association of serologic and protective responses of avian influenza vaccines in chickens. Avian disease 51: 481-483.

Laemmli UK (1970) Cleavage of structural proteins during the assembly of the head of bacteriophage T4. Nature 227: 680-685.

Liu W, Li H, Chen YH (2003) N-terminus of M2 protein could induce antibodies with inhibitory activity against influenza virus replication. FEMS Immunology and Medical Microbiology 35: 141-146.

Maas R, Tacken M, van Zoelen D, Oei H (2009) Dose response effects of avian influenza (H7N7) vaccination of chickens: serology, clinical protection and reduction of virus excretion. Vaccine 27: 3592-3597.

Park KS, Lee J, Ahn SS, Byun YH, Seong BL, Baek YH, Song MS, Choi YK, Na YJ, Hwang I, Sung YC, Lee CG (2009) Mucosal immunity induced by adenovirus-based H5N1 HPAI vaccine confers protection against a lethalH5N2 avian influenza virus challenge. Virology 395: 182-189.

Reed LJ, Muench H (1938) A simple method of estimating fifty per cent end points. American Journal of Epidemiology 27: 493-497

Rott R (1992) The pathogenic determinant of influenza virus. Veterinary Microbiology 33: 303-310.

Spackman E, Senne DA, Davison S, Suarez DL (2003) Sequence analysis of recent $\mathrm{H} 7$ avian influenza viruses associated with three different outbreaks in commercial poultry in the United States. Journal of virology 77: 13399-13402. 
Swayne DE, Beck JR, Garcia M, Stone HD (1999) Influence of virus strain and antigen mass on efficacy of H5 avian influenza inactivated vaccines. Avian Pathology 28: 245-255.

Swayne DE, Beck JR, Perdue ML, Beard CW (2001) Efficacy of vaccines in chickens against highly pathogenic Hong Kong H5N1 avian influenza. Avian Disease 45: 355-365.

Swayne DE, Garcia M, Beck JR, Kinney N, Suarez DL (2000a) Protection against diverse highly pathogenic H5 avian influenza viruses in chickens immunized with a recombinant fowlpox vaccine containing an $\mathrm{H} 5$ avian influenza hemagglutinin gene insert. Vaccine 18: 1088-1095.

Swayne DE, Lee CW, Spackman E (2006) Inactivated North American and European H5N2 avian influenza virus vaccines protect chickens from Asian H5N1 high pathogenicity avian influenza virus. Avian Pathology 35: 141-146.

Swayne DE, Perdue ML, Beck JR, Garcia M, Suarez DL (2000b) Vaccines protect chickens against H5 highly pathogenic avian influenza in the face of genetic changes in field viruses over multiple years. Veterinary microbiology 74: 165-172.

Swayne DE, Suarez DL, Spackman E, Jadhao S, Dauphin G, KimTorchetti M, McGrane J, Weaver J, Daniels P, Wong F, Selleck P, Wiyono A, Indriani R, Yupiana Y, Sawitri Siregar E, Prajitno T, Smith D, Fouchier R (2015) Antibody Titer Has Positive Predictive Value for Vaccine Protection against Challenge with Natural Antigenic-Drift Variants of H5N1 High-Pathogenicity Avian Influenza Viruses from Indonesia. Journal of Virology 89: 3746-3762.
Towbin H, Staehelin T, Gordon J (1979) Electrophoretic transfer of proteins from polyacrylamide gels to nitrocellulose sheets: procedure and some applications. Proceedings of the National Academy of Sciences of the United States of America 76: 4350-4354.

Van der Goot JA, Koch G, de Jong MC, vanBoven M (2005) Quantification of the effect of vaccination on transmission of avian influenza (H7N7) in chickens. Proceedings of the National Academy of Sciences of the United States of America 102: 18141-18146.

Van der Goot JA, Van Boven M, Stegeman A, van de Water SG, de Jong MC, Koch G (2008) Transmission of highly pathogenic avian influenza $\mathrm{H} 5 \mathrm{~N} 1$ virusin Pekin ducks is significantly reduced by a genetically distant $\mathrm{H} 5 \mathrm{~N} 2$ vaccine. Virology 382: 91-97.

Webby RJ, Perez DR, Coleman JS, Guan Y, Knight JH, Govorkova EA, McClain-Moss LR, Peiris JS, Rehg JE, Tuomanen EI, Webster RG (2004) Responsiveness to a pandemic alert: use of reverse genetics for rapid development of influenza vaccines. The Lancet 363: 1099-1103.

WHO (2002) WHO Manual on Animal Influenza Diagnosis and Surveillance 2nd 422 Edition. [Online.]

Wood JM, Kawaoka Y, Newberry LA, Bordwell E, Webster RG (1985) Standardization of inactivated H5N2 influenza vaccine and efficacy against lethalA/Chicken/Pennsylvania/1370/83 infection. Avian Disease 29: 867-872. 